\title{
EDITORIAL
}

\section{RELEVANCIA DE LA DIVULGACIÓN CIENTÍFICA PARA ENFERMERÍA EN LATINOAMÉRICA}

\section{Relevance of scientific disclosure of nursing in Latin America}

a Enfermería como ciencia, centra su responsabilidad investigativa en problemáticas relacionadas con el cuidado de las personas. Lo que se evidencia en las numerosas publicaciones indexadas en revistas de corriente principal, las cuales surgen de diversos esfuerzos investigativos, reflexivos y analíticos de profesionales de Enfermería inmersos/as tanto en la práctica como la academia a nivel mundial. Estos esfuerzos son valorados por la comunidad científica, especialmente cuando provienen de zonas geográficas como América del Norte y Europa. Si los hallazgos emergen desde América Latina es más trabajoso ser reconocido/a, sin embargo, la realidad investigativa actual ha tenido una evolución positiva.

Se destaca que la Enfermería Latinoamericana ha avanzado a pasos agigantados, en lo que a divulgación científica se refiere. De forma periódica, se originan nuevas formas de divulgación como, boletines y revistas en variados formatos, SANUS entre ellas. Sumado a que anualmente son más los congresos, simposios, coloquios, jornadas donde se pueden exponer, en modalidad oral o póster, avances de investigaciones, resultados de tesis y proyectos. Además, los programas de postgrado (especialidades, magíster y doctorados), distribuidos en países como México, Chile, Brasil, Colombia, Costa Rica, Ecuador, Argentina y Uruguay, aportan en la generación de nuevo conocimiento para fortalecer cada uno de los ámbitos de desempeño enfermero, entregando la posibilidad de generar redes multicéntricas potenciando los hallazgos investigativos de manera regional y no parcelada por países.

En este sentido y con la intención de fortalecer la ciencia de Enfermería, es que se exponen algunas propuestas para avanzar. La primera es que los profesionales de enfermería deben leer, citar y potenciar los estudios realizados por otras/os enfermeras/os, ya que muchas veces se priorizan otras ciencias versus la propia. Segundo, las enfermeras y enfermeros, deben atreverse a difundir los resultados de sus investigaciones o revisiones en revistas que no sean de Enfermería, de esta forma otros profesionales conocerán el aporte y la calidad científica de los estudios realizados. Tercera, los profesionales de enfermería deben trabajar sus propuestas investigativas de forma transdisciplinar. Y cuarto, enfermeros y enfermeras, deben utilizar los modelos y teorías de enfermería para sustentar sus investigaciones y el quehacer en la práctica.

Finalmente, se invita a las/los enfermeras/os de toda Latinoamérica a realizar un trabajo potenciador y de avance, facilitando los procesos divulgativos, sin dejar de lado la calidad científica de los hallazgos. Aportando en las revistas emergentes, como SANUS, para así contar con un gran abanico de posibilidades donde se puedan hacer públicos los resultados de los estudios en nuestra zona geográfica, respondiendo a aspectos culturales y de identidad, reconocidos en el leguaje y temáticas propias. Dichos aspectos permitirán a los futuros profesionales y a la sociedad en general, conocer el valioso aporte científico de la Enfermería en Latinoamérica e incrementar la relevancia que tiene la divulgación científica de enfermería para mejorar la calidad de los cuidados.

\author{
Paula Ceballos Vásquez \\ Doctora en Enfermería \\ Académica Departamento de Enfermería \\ Universidad Católica del Maule, Talca, Chile. \\ Contacto: pceballos@ucm.cl
}

